\title{
Magnitude of Common Childhood Illness, Health Care Seeking Behavior, and Associated Factors in Efratana Gidim District, East Amhara, Ethiopia, 2020
}

Genanew Kassie Getahun ( $\nabla$ genanaw21kassaye@gmail.com )

Kotebe Metropolitan University

Fentayehu Abebil

Public health professional specialist in ministry of mines and petroleum (MOMP)

Sindew Mahmud

Kotebe Metropolitan University

Ewunetu Firdawok

Addis Ababa University

Research Article

Keywords: Childhood illnesses, Healthcare seeking behavior, Ethiopia

Posted Date: September 8th, 2021

DOI: https://doi.org/10.21203/rs.3.rs-871754/v1

License: (c) (i) This work is licensed under a Creative Commons Attribution 4.0 International License.

Read Full License 


\section{Abstract \\ Background}

Childhood infectious illness mainly diarrheal diseases, febrile illnesses, and acute respiratory tract infection remains the leading cause of morbidity and mortality among children below five years. Delay and inappropriate healthcare-seeking behavior of caregivers' were the major reason for under-five child death in developing countries including Ethiopia. According to WHO, a timely healthcare-seeking practice can effectively save the lives of children by $20 \%$, particularly from ARIs, and significantly minimize morbidities. Therefore the aim of this study was to assess the magnitude of common childhood illness, healthcare-seeking behavior, and associated factors in Efratana Gidim District, East Amhara, Ethiopia, 2020.

\section{Methods}

A community based crossectional study was conducted from March 15 to April 15, 2020, among urban and rural respondents. Multistage sampling technique was employed with a total of 661 respondents by using semi-structured questionnaire through face to face interviews. Bivariate and multivariate logistic regression analyses were carried out to assess the association between healthcare-seeking behavior and predictor variables. Odds ratio along with $95 \%$ confidence interval was used to measure the strength of associations and statistical significance was considered at $p$-value $<0.05$.

\section{Results}

the overall two weeks prevalence of childhood illness was $24.1 \%$, (95\% Cl: $21.1 \%-27.3 \%)$ and $59.1 \%$, (95\% Cl: $51.1 \%-66.8 \%)$ of caregivers sought treatment at health facility. Moreover, fever, cough, and diarrhea accounted for $16.9 \%, 16.8 \%$, and $11 \%$ respectively. Caregivers' level of education (AOR $=2.56: 95 \% \mathrm{Cl}$ : 1.09 , $5.99)$ and residence $(0.26: 95 \% \mathrm{Cl}: 0.09,0.73)$ were significant factors for childhood illness and experience of child death (AOR $=3.766 ; 95 \% \mathrm{Cl}: 1.726,8.873)$, diarrheal symptoms $(\mathrm{AOR}=3.914 ; 95 \% \mathrm{Cl}: 2.043$, $10.828)$ and access to transportation $(A O R=3.352 ; 95 \% \mathrm{Cl}: 1.049,10.710)$ were predictors of healthcare seeking behavior of caregivers.

\section{Conclusion}

the prevalence of common childhood illness was high however; treatment-seeking behavior of caregivers for common childhood illness was low. Caregivers' experience of child death before, symptoms of diarrhea, and access to transportation were predictors of Healthcare-seeking behavior. Therefore repeated health education on basic prevention measures of common childhood illnesses and health promotion strategies to enhance caregivers' Healthcare-seeking behavior are critically important. . 


\section{Introduction}

Under-five child mortality is a core indicator not only for child health and well-being but also for the level of welfare in a country. Globally, under-five child mortality reduced from 93 deaths per 1,000 live births in 1990 to 38 in 2019(1). In Ethiopia, although significant achievements have been made under-five child mortality is still high accounting 55 deaths per 1000 live births (2) And the top leading causes were infectious diseases mainly diarrheal disease (25\%), pneumonia (21\%) and malaria (26\%)(3)

Common childhood illnesses are communicable diseases which is responsible for considerable underfive child morbidity and mortality particularly in low and middle-income countries across the world. Diarrheal disease, febrile illness and acute Respiratory infections are among the leading cause of childhood illnesses (4)

To reduce under-five child morbidity and mortality timely and appropriate healthcare-seeking behavior of caregivers' do have a significant contribution(5), despite that a large number of children still died before reaching health facilities due to delayed and inappropriate healthcare seeking(6). Hence WHO suggested that appropriate healthcare-seeking practice can effectively save the lives of children by $20 \%$, particularly from an acute respiratory infection(7).

Studies conducted in Ethiopia reported that healthcare-seeking behavior of caregiver were low and only few mothers sought treatment for their sick under-five children from health facility (8-10). According to Ethiopian Demographic Health Survey (EDHS) 2016 report, only 44\%, 35\%, and 30\% of caregivers sought treatment for diarrhea, fever, and acute respiratory infections respectively two weeks preceding the data collection period (11).

Different literature conducted elsewhere identified that caregivers' healthcare-seeking behavior for childhood illness were influenced by various factors including socio-demographic characteristics, accessibility to health services, severity of disease, distance to health facility and residence $(9,12,13)$.

In Ethiopia part of Sustainable Development Goals (SDGs), the government made significant strides to improve healthcare for childhood illness and to decrease death of under-five children with a minimum of 29 deaths per 1000 live births by 2020, even though the result was not as expected (14). A clear understanding on magnitude of common childhood illnesses, healthcare seeking behavior and determinant factors will lead to correct decision making by a caretakers and stakeholders who is vital to improve child health and healthcare delivery at all levels.

Despite the availability of different literature on the magnitude and determinant factors of healthcareseeking behavior of caregivers' for common childhood illnesses, evidences were not sufficiently available particularly with factors and magnitude of common childhood illnesses and some important factors of healthcare-seeking behavior like access to transportation and distance to health facility. Therefore, this study aimed to assess the magnitude of common childhood illness, healthcare-seeking behavior, and associated factors in Efratana Gidim District, East Amhara Ethiopia, 2020. 


\section{Objectives}

\section{General objective}

To assess the magnitude of healthcare-seeking behavior, common childhood illness, and factors associated with Healthcare seeking behavior in Efratana Gidim District, East Amhara, Ethiopia, 2020.

\section{Specific objectives}

1. To determine the magnitude of common childhood illnesses (Fever, Cough, and Diarrhea) in Efratana Gidim District, East Amhara, Ethiopia 2020.

2. To assess the magnitude of healthcare-seeking behavior of caregivers to their sick under-five children in Efratana Gidim District, East Amhara, Ethiopia 2020.

3. To identify factors associated with the healthcare-seeking behavior of caregivers in Efratana Gidim District, East Amhara, Ethiopia 2020.

\section{Methods}

\section{Study Area, design and period}

The study was conducted in Efratana Gidim District, North Shoa Zone, and Amhara regional state of Ethiopia. Efratana Gidim district (woreda) is one of the 27 woredas in North Shoa, which is $140 \mathrm{~km}$ far from Debre Birhan the capital of North Shoa Zone and $270 \mathrm{~km}$ far from Addis Ababa capital city of Ethiopia.

The total population of Efratana Gidim District was estimated to be 177,785 in 2019 , of which 85,791 were women and 24,079 were under-five children from the projection of 2007 Ethiopian census(15). There were 24 rural and 6 urban kebeles (the smallest administrative unit) and one primary hospital, 6 Health Centers (HC), 24 Health Posts (HP), and 20 private clinics.

The study was conducted from March 15 to April 15, 2020, with community-based cross-sectional study design.

\section{Source and study Population}

All caregivers who had children age between 2 and 59 months and residents of Efratana Gidim District were the source population.

Caregivers who had children age between 2 and 59 months and residents in the randomly selected kebeles of Efratana Gidim District at the time of the study were considered as the study population.

\section{Sample Size Determination}


The sample size was estimated using a single population proportion formula for the first objective considering the overall prevalence of childhood illnesses $26.5 \%(13)$, with a $5 \%$ margin of error and a $95 \%$ confidence level. The actual sample size was computed substituting the above values in the following formula:

$$
\begin{aligned}
& n=(\underline{Z a / 2}) \underline{\underline{2}} \underline{p}(\underline{1}-p) . \\
& d^{2} \\
& \mathrm{n}=(\underline{1.96}) \underline{\underline{2}} \underline{\underline{0.265}}(\underline{1-0.265}) . \\
& (0.05)^{2} \\
& n=299
\end{aligned}
$$

Where:

$\mathrm{n}=$ is required minimum sample size

$\mathrm{Za} / 2=$ critical value for $95 \%$ confidence level which is equals to $1.96(\mathrm{z}$ value at $\mathrm{a}=0.05$ )

$\mathrm{P}=$ overall prevalence of common childhood illnesses from a study conducted at Bahirdar, Ethiopia $d=$ an absolute precision margin of error $5 \%$.

The calculated sample size was 299 , and the required minimum sample of the study brings 329 caregivers having under-five children by adding a $10 \%$ non-response rate.

For the second objective by considering the magnitude of healthcare-seeking behavior of caregivers $48.8 \%$ (10), with a $5 \%$ margin of error and $95 \%$ confidence level.

Where:

$P=$ magnitude of healthcare-seeking behavior from the study conducted at Northwest Amhara, Ethiopia.

The calculated sample size was 384 , and the total required minimum sample size for the study brings 423 of caregivers having under-five children by adding a $10 \%$ non-response rate.

For the third objective by using double population proportion formula using Epi Info7 software for individual factors at $95 \%$ confidence level with $5 \%$ margin of error, $80 \%$ power and $1: 1$ ratio of exposed to unexposed were calculated.

Finally, by adding $10 \%$ possible non-response rate and design effect of 1.5 on the maximum sample size of the third objective the final sample size of the study was determined to be $\mathbf{6 7 4}$ caregivers' with underfive children. 


\section{Sampling Procedure}

Within Efratana Gidim district there were 30 Kebeles 24 rural and 6 urban. A multi-stage sampling technique was used to select participants for the study. All eligible kebeles were divided into two clusters; rural and urban. And then two urban and six rural kebeles were selected by lottery method randomly. The total sample size allocated to each eight kebeles were provided with proportional allocation to the number of under-five children per each selected kebeles.

Households having under-five children were identified by using a family folder from health posts and then households were selected with simple random sampling by lottery method using the household record from each health posts as a reference until the total sample size needed in each specific kebele was obtained.

Whenever there was two or more caregivers with under-five children in a family only one who were most intimate possibly biological mother of the selected under-five children were selected and if a caregiver has more than one under-five children within a household only one child was selected by lottery method.

\section{Data Collection Procedures and variables}

\section{Data Collection Tool}

Data was collected using semi-structured and pre-tested questionnaire. It was contextualized based on the research objective from the reviewed literature. To facilitate understanding of each question, first questionnaire were designed in English and then translated into Amharic, and translated back to English to check for consistency.

\section{Data Collection Procedure}

Informed voluntary written consent was obtained from each study participant after explaining the objective and purpose of the study. Data was collected through face to face interview by using semistructured questionnaire. It was collected by four clinical nurses and a public health officer was recruited for supervision.

\section{Dependent Variable}

Occurrence of common childhood illnesses with a child between 2 and 59 months (Cough, Diarrhea, and Fever)

Caregivers' healthcare-seeking behavior for their sick under-five children between 2 and 59 months.

\section{Independent Variables}

Socio-demographic factors: Caregivers age, educational status, marital status, family size, occupation, religion, child sex, child age and number of under-five children, 
Enabling factors: monthly income, distance from health facility, Experience of child death before, place of residence, access to transportation.

Predisposing factors: perceived severity of illness, perceived symptoms of illness.

\section{Operational Definitions}

Common childhood illnesses: includes acute respiratory infections, Diarrheal diseases, and febrile illnesses

Cough: was defined as the presence of cough which was reported by caregivers' within two weeks before the date of data collection.

Diarrhea: was defined as the passage of three or more loose/liquid stools per day as perceived and reported by caregivers' within two weeks before the date of data collection

Fever: were defined as a subjective feeling of increased body temperature as perceived and reported by caregivers' within two weeks before the date of data collection.

Healthcare-seeking behavior. Caregiver who responds for visiting health institutions for the recent symptom of his/her child with common childhood illness was considered as having healthcare-seeking behavior.

Presence of childhood illness: was the occurrence of at least one or more of the following symptoms (cough, diarrhea, or fever) within two weeks before the date of data collection.

Caregiver: was defined as a person who was responsible for taking care of a sick child and age 18 years and above; can be mother, father, or the relative of a child such as a guardian or legally approved person by the court.

Access to Transportation: was defined as the ability of caregivers to get safe and affordable transportation vehicles within five-kilo meters of the radius.

\section{Data Quality Assurance}

Data quality was assured through training of data collectors and a supervisor before a date of data collection. Pilot testing was done at Kewot District, near to Efratana Gidim district and had similar sociodemographic characteristics, which were done with $5 \%$ of the sample size and were finally double entered to compare and assure the quality of the data.

\section{Data Processing and Analysis}

Data entry was made using Epi-data version 3.1 and then exported to Statistical Package for Social Sciences (SPSS) version 26 for further analysis. Before analysis, data were cleaned for possible errors 
and completeness and consistencies of questionnaires were checked. Descriptive statistics was made for categorical and continuous variables in the form of percentage and means respectively.

Binary logistic regression model were carried out to identify variables that could independently associate with healthcare-seeking behavior. Variables whose p-value less than 0.25 in bi-variable analysis were included in the multivariable model. Odds ratio along with 95\% confidence level were estimated to measure the strength of association. Variables were interpreted as having a statistically significant association when the p-value was $<0.05$.

\section{Results}

\section{Demographic and socio-economic characteristics of respondents}

A total of 661 caregivers' were interviewed giving an overall response rate of $98 \%$, and the majority $92.6 \%$ of them was married. Almost all, $99.1 \%$, of the study participants were mothers as primary caregivers.

The mean ( $\pm S D)$ age of caregivers' was $37.44( \pm 7.29)$ with a range of 21 to 68 years. Most of the respondents (84.6\%) were in the age group of $25-45$ years and all most all $623(94 \%)$ of participants were Orthodox by religion.

Regarding the occupational status of respondents, the majority $473(71.6 \%)$ were housewives followed by government-employed (19\%), and nearly two-thirds of the respondents $419(63 \%)$ had only one under-five child.

With regard to the sex of the child nearly half 333 (50.4\%) were males and the rest $49.6 \%$ were females. The mean $( \pm S D)$ age of children was $24.19( \pm 13.3)$ months ranging from 2-58 months. Two hundred seventy four (41.5\%) of them were within the age group of $25-36$ months.

The median monthly income of respondents were 900 birr with the range of 100 to 8000 birr per month and only $306(46.3 \%)$ of respondents have attended formal education.

Table 1: Demographic and socio-economic characteristics of study participants in Efratana Gidim district, east Amhara, Ethiopia, 2020 (n=661). 


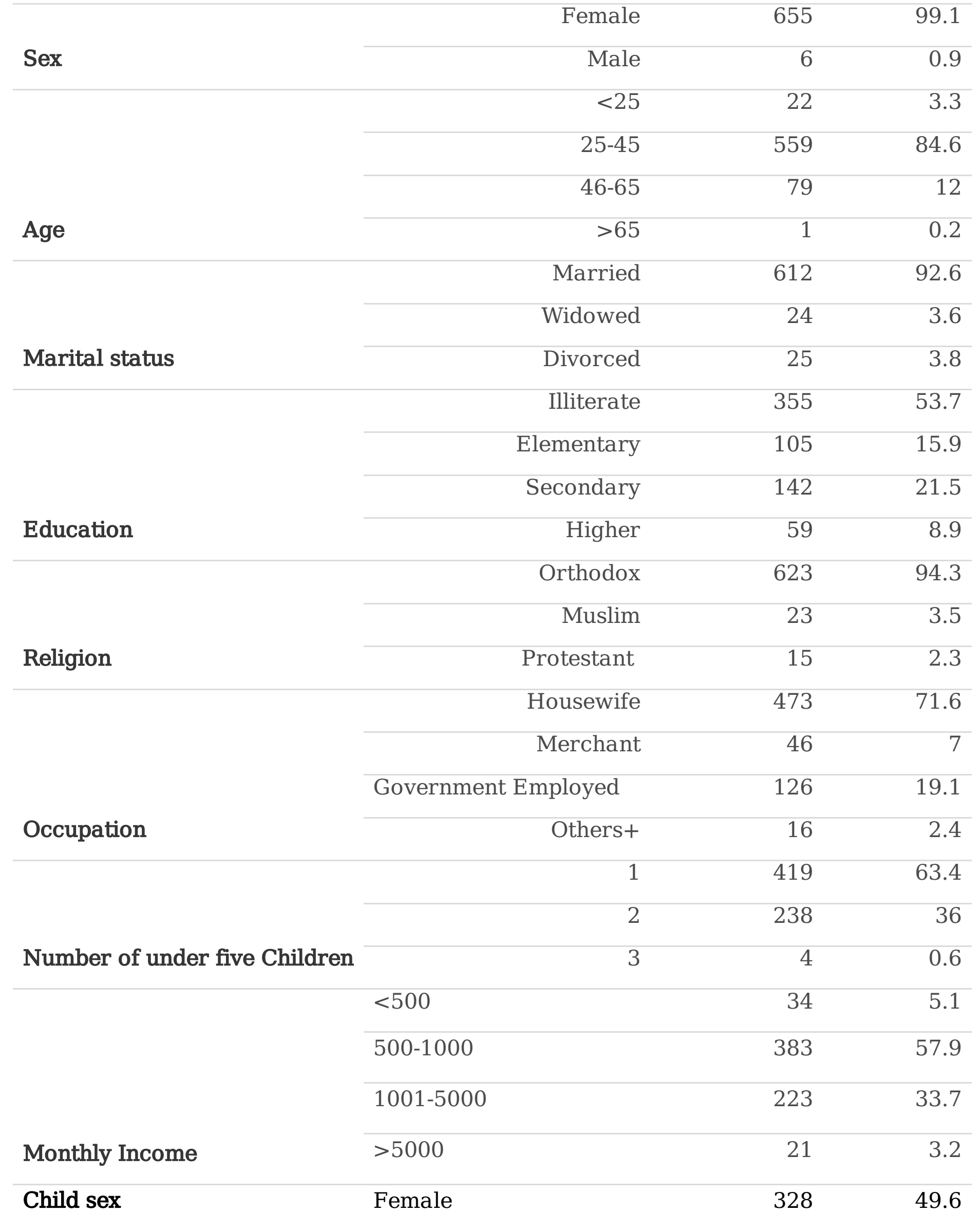




\section{3-24 months}

25-36 Months

\section{Child Age}

37-59 Months
88

13.3

274

41.5

96

Others+ includes Farmers and Daily laborers

\section{Enabling and need related characteristics of respondents}

Of the total 661 participants responded the average (mean) household size was four in numbers and nearly one third 209 (31.6\%) of the respondents were urban residents.

Regarding distance from the health facility and access to transport one-fourth of respondents $161(24.4 \%)$ were living more than five-kilo meters far from health institutions, meanwhile, the majority of $302(45.7 \%)$ respondents reported that they did not have any access to transportation services.

Out of the total study, participants interviewed only 38 (5.75\%) reported that the household had an experience of child death before the time of interview and only two percent of participants had a sick child with severe disease as perceived by the respondents.

Table 2: Enabling and need related factors of respondents in Efratana Gidim district, east Amhara, Ethiopia, $2020(\mathrm{n}=661)$. 


\begin{tabular}{llrr} 
Variables & Category & Frequency (n) & Percent (\%) \\
\hline \multirow{2}{*}{ Distance from Health Facility } & $<=5 \mathrm{~km}$ & 500 & 75.6 \\
\cline { 2 - 4 } & $>5 \mathrm{~km}$ & 161 & 24.4 \\
\hline Residence & Rural & 452 & 68.4 \\
\hline \multirow{2}{*}{ Family Size } & Urban & 209 & 31.6 \\
\hline \multirow{2}{*}{ Severity of disease } & $<=5$ & 546 & 82.6 \\
\cline { 2 - 4 } & $>5$ & 115 & 17.4 \\
\hline \multirow{2}{*}{ Experience of Child death } & Severe & 13 & 2 \\
\cline { 2 - 4 } & None severe & 648 & 98 \\
\hline & No & 38 & 5.75 \\
\hline & Yes & 623 & 94.25 \\
\hline Access to Transportation & Sometimes & 209 & 31.6 \\
\cline { 2 - 4 } & No & 150 & 22.7 \\
\hline
\end{tabular}

\section{Childhood illness prevalence}

The overall prevalence of common childhood illnesses which have one or more symptoms among underfive children between two and fifty-nine months was $24.1 \%$, (95\% Cl: $21.1 \%-27.3 \%)$. Out of 661 households surveyed, presence of cough, diarrhea and fever were $16.8 \%,(95 \% \mathrm{Cl}$ : 14\%-19.9\%), 11\%, (95\% Cl: $8.8 \%-13.7 \%)$ and $16.9 \%,(95 \% \mathrm{Cl}: 14.2 \%-20 \%)$ respectively.

Furthermore, $2.4 \%$, (95\% Cl: $1.4 \%-3.9 \%)$ of children between the age of 2-59 months had symptoms of cough, fever, and diarrhea simultaneously.

\section{Healthcare Seeking Behavior}

Overall 59.1\%, (95\% Cl: $51.1 \%-66.8 \%$ ) of respondents sought healthcare for their sick child. Out of 94 caregivers who have sick children and sought modern medical treatment the most $28.7 \%$ of respondent's primary preference were health institutions followed by traditional healers $17 \%$, holy water $16 \%$, religious institutions $13.8 \%$, at home $12.8 \%$ and from pharmacy $11.7 \%$. For caregivers who preferred health facilities, health centers were the most chosen source of healthcare services where treatment was chosen 
by $50 \%$ of caregivers and the rest $35.1 \%$ sought treatment from health posts, $8.5 \%$ from the hospital, and $6.4 \%$ from a private clinic. The major reasons for treatment-seeking from health facilities were the child's condition worsened $51.1 \%$ followed by fear of complication $26.6 \%$, people's advice $18.1 \%$, and facility was nearly $4.3 \%$.

Table 3: Caregivers healthcare seeking behavior for common childhood illnesses in Efratana Gidim district, east Amhara, Ethiopia, 2020.( $n=159)$ 


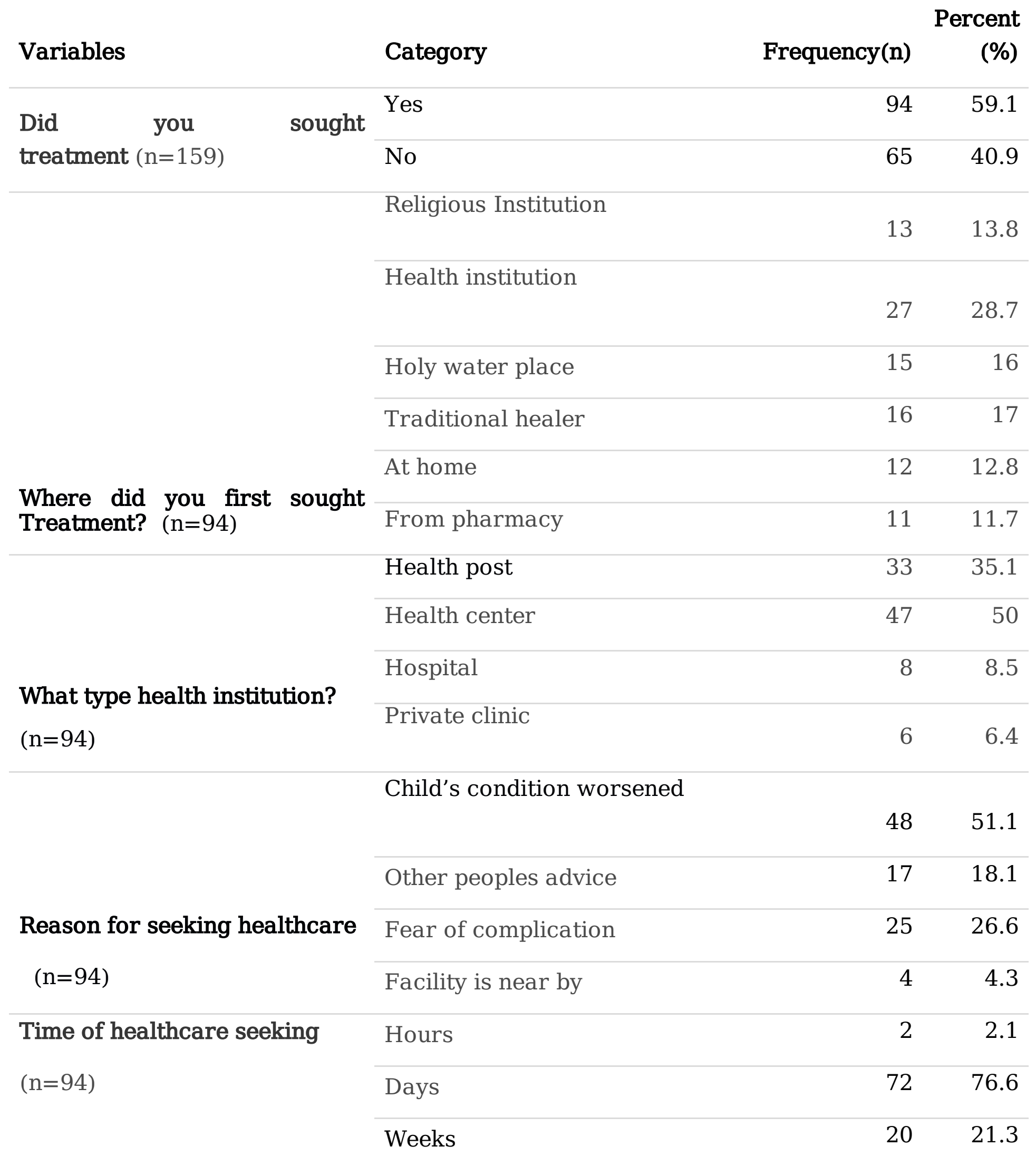


symptom recognition and $20(21.3 \%)$ were sought treatment weeks after the onset of symptoms and very few caregivers $2(2.1 \%)$ were sought treatment immediately within hours of symptom recognition.

As reported by participants the main reason for not seeking healthcare were identified and mentioned as treatment cost being expensive 19 (29.2\%) followed by illness was not serious 16 (24.6\%), lack of transportation $13(20 \%)$, lack of money 11 (16.9\%), and long-distance from health facility $6(9.2 \%)$.

\section{Factors affecting common childhood illnesses}

In the bivariate analysis caregivers' level of education, religion, occupation, monthly income, residence of caregiver, number of under-five children, and child age were associated with common childhood illness.

However religion of caregiver, occupation, monthly income, and number of under-five children were not significantly associated with childhood illness by multivariable logistic regression analysis.

Meanwhile level of education ( $A O R=2.56: 95 \% \mathrm{Cl}: 1.09,5.99)$ and caregivers' residence $(A O R=0.26: 95 \% \mathrm{Cl}$ : $0.09,0.73$ ) were determinant factors of common childhood illnesses at $p$ value $<0.05$.

Table 4: Factors affecting childhood illness in Efratana Gidim district, Ethiopia, 2020 $(\mathrm{n}=661)$ 


\section{No Yes}

\section{Educational Status}

Illiterate

Elementary

Secondary

Higher

16

16
263

$1.06(0.57,1.98)$

$0.96(0.34,2.75)$

$34 \quad 71$

$0.78(0.38,1.57)$

$0.81(0.27,2.48)$

125

$2.74(1.27,5.88)$

$2.56(1.09,5.99)^{*}$

\section{Religion}

Orthodox

Muslim

$145 \quad 478$

1.65(0.55, 4.90)

$3.21(0.92,11.24)$

Protestant

$9 \quad 14$

$0.78(0.19,3.04)$

$0.67(0.15,2.93)$

Occupation

Housewife

5

10

1

1

Merchant

125348

$0.58(0.22,1.55)$

$0.82(0.26,2.58)$

Government Employed

$22 \quad 104$

$0.77(0.22,2.77)$

$0.82(0.19,3.45)$

Others

5

24

1

$0.99(0.34,2.87)$

$0.49(0.13,1.83)$

\section{Number of under-5 children}

$\begin{array}{lllll}1 & 98 & 321 & 0.95(0.65,1.38) & 8.02(0.70,91.88) \\ 2 & 58 & 180 & 0.11(0.01,0.99) & 8.30(0.72,95.37) \\ 3 & 3 & 1 & 1 & 1\end{array}$

\section{Average monthly income}

$<500$

10

22

$0.52(0.14,1.94)$

$0.49(0.11,2.41)$

500-1000

$106 \quad 279$

$0.62(0.21,1.88)$

$0.79(0.19,3.24)$ 

1001-5000
$39 \quad 184$
$1.11(0.35,3.48)$
$1.21(0.35,4.14)$
$>5000$
4
17
1
1

\section{Residence}
Urban
33
181
$2.06(1.35,3.15)$
$0.26(0.09,0.73)^{* *}$
Rural
122
321
1
1

\section{Child Age}

2-12 Months

13-24 Months

25-36 Months

37-59 Months

20

76

142

$0.61(0.34,1.09)$

$0.86(0.44,1.65)$

20

68

$0.89(0.44,1.81)$

$1.13(0.52,2.45)$

$58 \quad 216$

$0.98(0.55,1.74)$

$1.23(0.65,2.33)$

1

*significant at $(\mathrm{P}<0.05), * *$ significant at $(\mathrm{P}<0.01), * * *$ significant $(\mathrm{P}<0.001)$

\section{Determinant factors associated with healthcare-seeking behavior}

The result of bivariate analysis revealed that educational status of caregiver, caregivers occupation, number of under-five children, monthly income, distance to the health facility, household residence, child age, a caregiver who had an experience of child death before, presence of diarrheal symptoms, presence of cough, diarrhea, and fever together and accesses of transportation were significantly associated with healthcare-seeking behavior and were a candidate for multivariable logistic regression, whereas the age of caregiver, marital status, household size, caregivers religion, sex of caregiver, presence of cough symptom, child sex and perceived severity of childhood illness by caregivers were not significant at $p$ value $<0.25$.

Moreover, the result of multivariable logistic regression analysis showed that experience of child death before [AOR=3.766; 95\% Cl: 1.726, 8.873], presence of diarrheal symptom [AOR=3.914; 95\%Cl: 2.043 , 10.828] and accesses to transportation [AOR=3.352; $95 \% \mathrm{Cl}: 1.049,10.710]$ were independent predictors of healthcare-seeking behavior of caregivers for common childhood illnesses. However, caregivers level of education, caregivers occupation, household monthly income, distance from a health facility, place of 
residence, number of under-five children, child age and presence of cough, diarrhea and fever symptoms together were not significantly associated with healthcare-seeking behavior of caregivers for common childhood illnesses in the multivariable analysis at $p$-value $<0.05$.

Table 5: Factors associated with healthcare seeking behavior in Efratana Gidim woreda, east Amhara, Ethiopia, 2020. ( $\mathrm{n}=159)$ 


\section{Yes No}

\section{Educational Status}

$\begin{array}{lcccl}\text { Illiterate } & 48 & 44 & 1.058(0.481,2.328) & \begin{array}{l}1.136(0.440, \\ 2.936)\end{array} \\ \text { Elementary } & 18 & 16 & 0.159(0.034,0.734) & \begin{array}{l}0.218(0.024, \\ 1.936)\end{array} \\ \text { Secondary } & 15 & 2 & 0.275(0.073,1.029) & \begin{array}{l}0.233(0.018, \\ 3.046)\end{array} \\ \text { Higher } & 13 & 3 & 1 & 1\end{array}$

\section{Occupation}

Housewife

$70 \quad 55$

$0.636(0.182,2.224)$

0.626(0.112, 3.506)

Merchant

$6 \quad 6$

$0.283(0.090,0.884)$

0.452(0.045, 4.502)

Government Employed $\quad 18$

4

\section{Number of under- 5 children}

$\begin{array}{rrrrr}1 & 53 & 45 & 0.576(0.290,1.141) & \begin{array}{r}0.439(0.177, \\ 1.084)\end{array} \\ & & & \\ & & & & \\ & 40 & 18 & 2.558(0.224,29.156) & 2.934(0.155, \\ & & & 55.627)\end{array}$

$\begin{array}{lll}1 & 2 & 1\end{array}$

1

\section{Average monthly income}

$\begin{array}{rrrrl}<500 & 7 & 3 & 2.006(0.492,8.178) & \begin{array}{l}1.909(0.345, \\ 10.561)\end{array} \\ 500-1000 & 55 & 51 & 0.805(0.174,3.722) & \begin{array}{l}0.681(0.077, \\ 6.034)\end{array} \\ 1001-5000 & 29 & 10 & 0.778(0.056,10.861) & \begin{array}{r}3.398(0.102, \\ 113.41)\end{array}\end{array}$


$>5000$

3

1

1

1

Distance

$<5 \mathrm{~km}$

74

44

$1.90(0.926,3.90)$

1.084(0.441,

2.663)

$>5 \mathrm{~km}$

20

21

1

1

\section{Residence}

Urban

26

7

2.971(1.201, 7.349)

0.175(0.009,

3.441)

Rural

68

58

1

1

\section{Child Age}

2-12 Months

36

25

$0.830(0.290,2.379)$

1.087(0.281, $4.212)$

13-24 Months

13

7

$0.811(0.385,1.711)$

0.932(0.377,

2.305)

25-36 Months

$38 \quad 20$

2.312(0.824, 6.488)

3.624(0.994,

13.209)

37-59 Months

7

13

1

1

\section{Experience of child death}

Yes

$$
30
$$

5

5.625(2.048, 15.446)

3.766(1.726, 8.873)*

No

64

60

1

1

\section{Diarrhea}

Yes

53

19

3.130(1.598,

6.130)

3.914(2.04, 10.83)***

No

41

46

1

1 


\section{Cough, Diarrhea and Fever}
Yes
13
3
3.133(0.855,
11.478)
$1.396(0.233,8.343)$

No

81

62

1

1

\section{Access of Transportation}

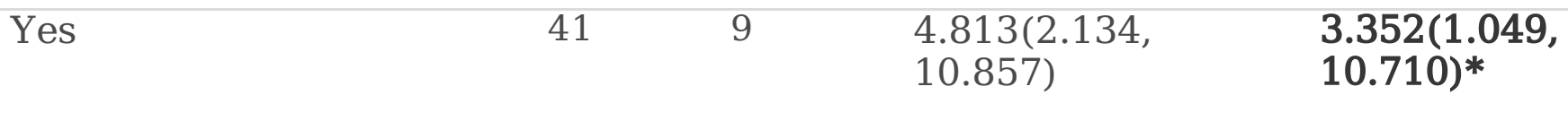

$\begin{array}{lllll}\text { No } & 53 & 56 & 1 & 1\end{array}$

*significant at $(\mathrm{P}<0.05), * *$ significant at $(\mathrm{P}<0.01), * * *$ significant at $(\mathrm{P}<0.001)$

\section{Discussion}

In this study, the overall prevalence of common childhood illness of under-five children between 2 and 59 months was $24.1 \%$, (95\% Cl: $21.1 \%-27.3 \%)$. Of these $16.9 \%$ were fever, $16.8 \%$ were cough and $11 \%$ were diarrhea. Which was high compared with the national figure EDHS 2016 report (11). However, this finding is lower than studies conducted at Addis Ababa Ethiopia, Tanzania, and India $(9,16-18)$. The discrepancy might be due to the difference in the socio-demographic characteristics, the time of the study, and methods of data collection. For example, a study conducted in Addis Ababa Ethiopia was a facility-based study done in 2016 and the study populations were caregivers with sick under-five children only which might increase the prevalence proportional to all sick child and another study done at Tanzania were conducted at the most rural districts with a high burden of common childhood illness as well as poor access and utilization of healthcare services which have a potential to increase the prevalence of common childhood illness.

Similarly, the two weeks period prevalence of common childhood illnesses was low compared with studies conducted in different parts of the Amhara region(19-21). The difference in such prevalence could be due to the study period, sample size, and was primarily focused on diarrheal illnesses. For instance, a study conducted in Farta district North West Ethiopia was conducted in 2014 and focused mainly on diarrhea and it was done with respondents from only rural kebeles of the district where a child can acquire any illness due to poor access to basic healthcare services like immunization might contribute to high prevalence.

Further caregivers' level of education and caregivers' residence were significant factors of common childhood illnesses. 
Caregivers' level of education was significantly associated with childhood illness after controlling other socio-demographic and enabling factors. In such a caregiver with secondary education were 2.5 times more likely to have a child with childhood illness compared with higher educated caregiver. This finding is supported by previous studies done at Jimma, Ensaro, and EDHS 2016 analysis(22-24). This might be due to the fact that more educated caregivers' could have knowledge and adequate information about prevention mechanism of childhood illness and can easily comprehend with health messages.

Occurrences of childhood illness were decreased by $74 \%$ among urban caregivers' compared with rural caregivers. This finding was consistent with other study findings(12,21). This might be due to the difference with socio-economic status and infrastructures like access to water supply and provision of latrine facility.

The current study revealed that the healthcare-seeking behavior of caregiver for common childhood illness was $59.1 \%$, (95\% Cl: $51.1 \%-66.8 \%)$. Of 159 children with one or more symptoms, their respective caregivers sought treatment for $53(73.6 \%)$ of diarrhea, $68(61.8 \%)$ of fever, and $63(57.8 \%)$ of cough. Which is in agreement with a study conducted at Ensaro District North Shoa 59.9\%, Kenya Nairobi slums $60.5 \%$ and Rwanda 58.6\% $(17,23,25)$. However, it is higher than the EDHS 2016 report and studies conducted in Addis Ababa and northwest Ethiopia (9-11). This might result from differences in study setting, differences in the study period, and sample size. The study of Northwest Ethiopia particularly in the Aneded district was conducted in 2016 and only rural mothers participated for the interview, which could reduce their healthcare-seeking behavior due to poor awareness, distance, and poor access to the health facilities.

In contrast, this finding is lower than previous similar studies conducted in different regions of Ethiopia (13,26-28). The possible explanations for this might be due to some of the above studies were done in urban settings which can have access to healthcare and quality of services near to their household and in the other way, most of the study was primarily focused on diarrheal disease which could be perceived by caregivers' as more sever and they might seek treatment better for their sick child.

The current study also identified main reasons reported by caregivers' for not seeking treatment from health facilities and the major reasons were caregivers' perception that the illnesses were not serious $(24.6 \%)$, expensiveness of treatment $(29.2 \%)$, and lack of transportation (20\%). These might be due to rural caregivers' limited awareness to recognize illness severity and when to seek treatment in addition to limited resources and poor economic status.

Findings from multivariate logistic regression analysis showed that experience of child death before, presence of diarrheal symptoms, and access to transportation were independent predictors of healthcareseeking behavior of caregivers for common childhood illnesses.

The caregiver's previous experience of child death was a strong predictor of healthcare service utilization. The healthcare-seeking behavior of caregivers with experience of child death before was nearly four times the odds to utilize healthcare services than their counterparts. This finding was in line with a study finding 
from Addis Ababa, southwest Ethiopia, and Nigeria $(9,29,30)$. This might be due to the fact that previous bad experience of child death might motivate caregivers to recognize danger signs and symptoms of an ill child that is necessary to take immediate action and appropriate management for disease conditions, which can also reduce complication and deaths. On the other way, a caregiver with experience of child death before could be more sensitive to save the life of the rest live children.

Treatment was sought better for children with diarrhea as compared to cough and fever. The sick underfive children with symptoms of diarrhea were four times the odds to utilize healthcare than symptoms of cough and fever. This finding was comparable with the finding reported from different studies $(13,30,50)$. This could be due to the fact that rural caregivers might consider diarrheal illnesses as more severe than symptoms of cough and fever since diarrhea is more visible for caregivers than cough or fever. On the other hand, it could be due to the fact that the availability of effective treatment that can be safely administered at home and provision of information to the rural community about the treatment services.

Moreover, the odds of healthcare-seeking behavior of caregiver having access to transportation were nearly three times more utilized healthcare services compared with caregivers who did not have any transport access. This might be justified by children living in the most rural communities far from health institutions without access to transportation were denied of access to treatment services for common childhood illnesses which could lead to increased occurrence of childhood morbidity and mortality. In the other hand caregiver who has access to transportation might have better socioeconomic status and good attitude to seek modern treatment for their sick under-five children and can better comprehend to health messages and choose cost-effective interventions.

With the current study, it was noted that factors like age of caregiver, marital status, occupation, level of education, religion, age and sex of the sick child, household income, residence, distance from the health facility and perceived severity of illness, did not show significant association with healthcare-seeking behavior. The reason for this might be due to the fact that most of the respondents were housewives and married, had no formal education and most of caregivers sought treatment from health facilities when the diseases get worsened.

\section{Strengths and limitations of the study}

Strength: Being a community-based study and minimal none response rate.

\section{Limitation}

Measurement of common childhood illness was not validated clinically since it was taken only by caregivers' perception of common childhood illness. Since the data was collected based on self-report of caregivers' healthcare-seeking, thus might be susceptible to recall, reporting, and social desirability bias. Finally, hence a cross-sectional study design was used the association might not imply temporal relationship. 


\section{Conclusions And Recommendations}

\section{Conclusions}

In conclusion, the two weeks prevalence of common childhood illness was high compared with the national figure EDHS 2016 report(11) and, treatment-seeking behavior of caregivers' for common childhood illness was low compared with some studies conducted in the Amhara region(32,33).

Most caregivers sought treatment from health facilities after worsening of common childhood illness manifestations. Furthermore, caregivers' level of education and residence were significant factors for common childhood illness and experience of child death before, symptoms of diarrhea, and access to transportation were independent predictors of healthcare-seeking behavior of caregivers' for common childhood illnesses.

\section{Recommendations}

Based on findings from the current study I would like to suggest the following,

Efratana Gidim District health office; to enhance the provision of basic preventive measures like immunization and repeated health education about proper housing, hygiene, and sanitation which helps to reduce child morbidity and mortality and to improve the health status of under-five children.

The office should also strengthen infrastructures for transport access and health facility for the most neglected rural population.

Healthcare professionals: should provide health education to enhance caregivers' awareness about childhood illnesses and to promote healthcare-seeking practices of caregivers by using IEC/BCC materials.

\section{List Of Abbreviations}

The following are some of the key abbreviations used in this study. ARI - Acute Respiratory tract Infection, BCC-Behavioral Change Communication, EDHS-Ethiopian Demographic and Health Surveys, GBD-Global Burden of Disease, HSDP-Health Sector Development Program, ICCM-Integrated Community Case Management, IEC-Information Education and Communication, PHC-Population and Housing Census, SDGs-Sustainable Development Goals, SPSS-Statistical package for social sciences, UNICEF-United Nations International Children's Emergency Fund-WHO-World Health Organization

\section{Declarations}

\section{Ethical consideration}


Ethical approval was obtained from Addis Ababa University, College of Health Science Ethical Review Board (IRB). Permission letter was obtained from all local administrators and health managers. An informed consent was obtained from mothers/care givers. All study participants were informed that the participation was voluntarily. The potential benefits, harms, confidentiality, and the possibility of withdrawing from the interview at any time of interview and after were also informed.

\section{Consent for publication}

Not applicable

\section{Data Availability}

The data used to support the findings of this study are available from the corresponding author upon request.

\section{Conflicts of Interest}

The author declares they have no competing conflicts of interest.

\section{Funding}

The study has no funding source.

\section{Acknowledgement}

The authors acknowledge all mothers/caregivers of children for their cooperation and willingness. We would also like to thank data collectors for their great collaboration and tolerance for this research work

\section{References}

1. UN Inter-agency Group for Child Mortality Estimation, UNICEF, WHO, WBO. Levels \& Trends in Child Mortality Estimation Child Mortality [Internet]. Un Igme. 2020. 48 p. Available from: https://www.unicef.org/media/79371/file/UN-IGME-child-mortality-report-2020.pdf.pdf

2. Institute E public health, ICF. Mini Demographic and Health Survey: key indictors [Internet]. Handbook of Federal Countries, 2005. 2019. 136-148 p. Available from: www.DHSprogram.com.

3. Johnson HL, Liu L, Fischer-Walker C, Black RE. Estimating the distribution of causes of death among children age 1-59 months in highmortality countries with incomplete death certification. Int $\mathrm{J}$ Epidemiol. 2010;39(4):1103-14.

4. Kebede ZT. High common childhood illnesses while treatment-seeking behavior of care givers' was low. Res Sq. 2019;1-22.

5. Adedire Elizabeth A et al. Maternal Perception and HealthCare-Seeking behaviors for Childhood Febrile Illnesses in Rural Communities of Osun State, Nigeria. Sci J Public Heal. 2015;2(6):636.

6. Estimation CM. levels and trends in Child Mortality 2017. WHO. 2017;15-6. 
7. Victora CG, Bryce J, Fontaine O, Monasch R. Reducing deaths from diarrhoea through oral rehydration therapy. Bull World Health Organ. 2000;78(10):1246-55.

8. Alene M, Yismaw E. Healthcare utilization for common childhood illnesses in rural parts of Ethiopia: Evidence from the 2016 EDHS. BMC Public Health. 2019;19(1):1-12.

9. Abegaz NT. Caregivers healthcare seeking behavior towards childhood illness in selected health centers in Addis Ababa, Ethiopia: BMC Pediatr. 2019;19(1):1-9.

10. Simieneh MM, Mengistu MY, Gelagay AA, Gebeyehu MT. Mothers' health care seeking behavior and associated factors for common childhood illnesses, Northwest Ethiopia: Community based crosssectional study. BMC Health Serv Res. 2019;19(1):1-7.

11. Survey H. Ethiopia. Rockville, editor. Maryland, USA. CSA and ICF 2016; 2016.

12. Tsion, Assefa T, et al B. Assefa T,Belachew T. et al.caregivers' HealthCare Seeking Behavior for common Childhood Illnesses in Derra District, Ethiopia. Ethiop J Heal Sci;2008; 2008;18(3):(6):8794.

13. Worku A. Prevalence of common childhood illness and caregivers ' healthcare seeking behavior in Bahir Dar, Ethiopia: J Prev Med. 2013;3(2):155-9.

14. Ethiopian Federal Ministry of Health. Health Sector Transformation Plan (2015/16-2019/20). 2015;20(May):1-118.

15. CSA. 2007 Population and Housing Census of adminstrative,. 2012;(April):125.

16. Yamuna B. Prevalence of acute morbidities and health seeking behaviour among under-five children of urban slums in Karnataka. 2017;4(7):2449-56.

17. Taffa, Chepngeno $\mathrm{G}$ et al. Determinants of healthcare seeking for childhood illnesses in Nairobi. Trop Med Int Heal. 2005;10(3):240-5.

18. Kanté AM eatal. Prevalence of common childhood Illness and Healthcare Seeking Behavior of caregivers' in Rural Tanzania. BMC Public Health [Internet]. 2015;15:951:1-12. Available from: http://dx.doi.org/10.1186/s12889-015-2264-6

19. Azage M, K. Childhood diarrheal disease in high and low hotspot districts of Amhara Region, Ethiopia. J Heal Popul Nutr [Internet]. 2016;35:13:1-14. Available from: http://dx.doi.org/10.1186/s41043-016-0052-2

20. Gedefaw M, Moges.etal. Diarrheal Diseases and determinant factors in the Rural Dejen District, Ethiopia, 2014. Am Sci Res J. 2015;5: 1,(January 2014):1-13 [3].

21. G.etal G. Magnitude and Associated Factors of Diarrheal disease among Children below five years in Farta District, Ethiopia. Qual Prim Care. 2017;25 (4)(January):199-207.

22. Shaw B, Amouzou A, P M. Determinants of Utilization of Health Extension Workers in the Context of Scale-Up of ICCM of Childhood Illnesses in Ethiopia,. Am Soc Trop Med. 2015;93(3):636-47.

23. Sisay S, Endalew G, Hadgu G. Assessment of HealthCare Seeking Behavior for Childhood Illness in Rural Ensaro District, Ethiopia 2014. Res gate. 2017;(May 2016). 
24. et al TM. Factors determining under-five children with symptoms of acute respiratory tract infection in Ethiopia: based on the 2016 DHS. 2018;

25. Sciences H. Healthcare seeking behavior of caregivers for sever respiratory infections,. 2017;

26. Fissehaye T, et al D. Healthcare seeking behaviour of caregivers towards diarrheal disease of underfive children in Mekelle city, Ethiopia. BMC Res Notes [Internet]. 2018;11:749:1-7. Available from: https://doi.org/10.1186/s13104-018-3850-3

27. Demissie B, Demeke HZ, International P, Sagaro G, Haidar J. The Ethiopian Of Health Development Joint Scholarly Publication of the Ethiopian Public Health Association and the School of. 2014; (January).

28. Kolola T, Gezahegn T, Addisie M. Health care seeking behavior for common childhood illnesses in jeldu district, oromia regional state, Ethiopia. PLoS One. 2016;11(10):1-11.

29. Getahun A, D. Determinant factors of delay in malaria healthcareabd-seeking practice for children below five years in south-west Ethiopia: Malar J. 2010;9(1):1-6.

30. Abdulraheem I eta. Determinant factors for caregivers' treatment-seeking behaviour for childhood illnesses in a rural Nigerian. Early Child Dev Care. 2009;179(5):671-83.

31. Gelaw YA, Biks GA, Alene KA. Effect of residence on mothers ' health care seeking behavior for common childhood illness in Northwest Ethiopia: a community based comparative cross - sectional study. 2014;1-8.

32. Gelaw YA. Effect of residence on caregivers healthcare-seeking practice for childhood illness in Northwest Ethiopia: BMC Res Notes. 2014;7(1):1-8.

33. Dagnew AB, Tewabe T, Murugan R. Level of modern health care seeking behaviors among mothers having under five children in Dangila town, north West Ethiopia, 2016 : a cross sectional study. 2018;1-6.

\section{Figures}




\section{Clinical characteristics of the sick child}

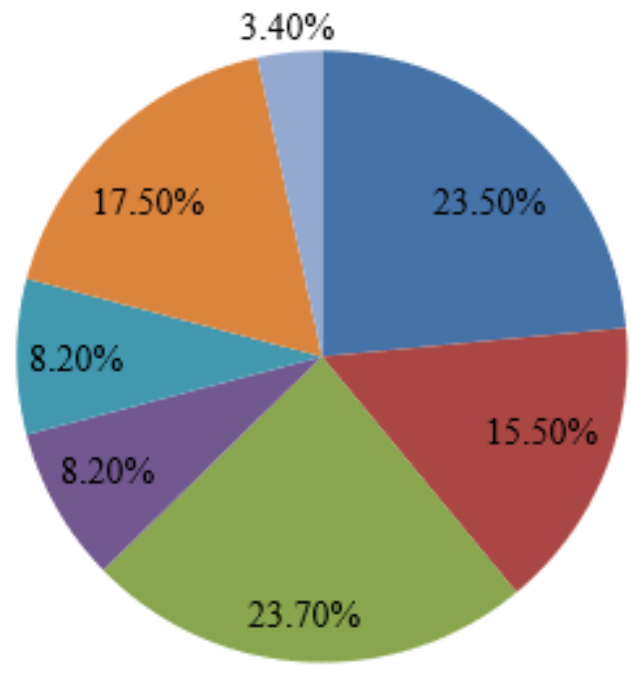

- Cough only

Diarrhea only

Fever only

a cough and Diarrhea

Diarrhea and Fever

- Cough and Fever

Cough, Diarrhea and Fever

Figure 1

Clinical characteristics of sick under-five children between 2 and 58 months in Efratana Gidim district, east Amhara, Ethiopia, 2020 ( $n=159)$.

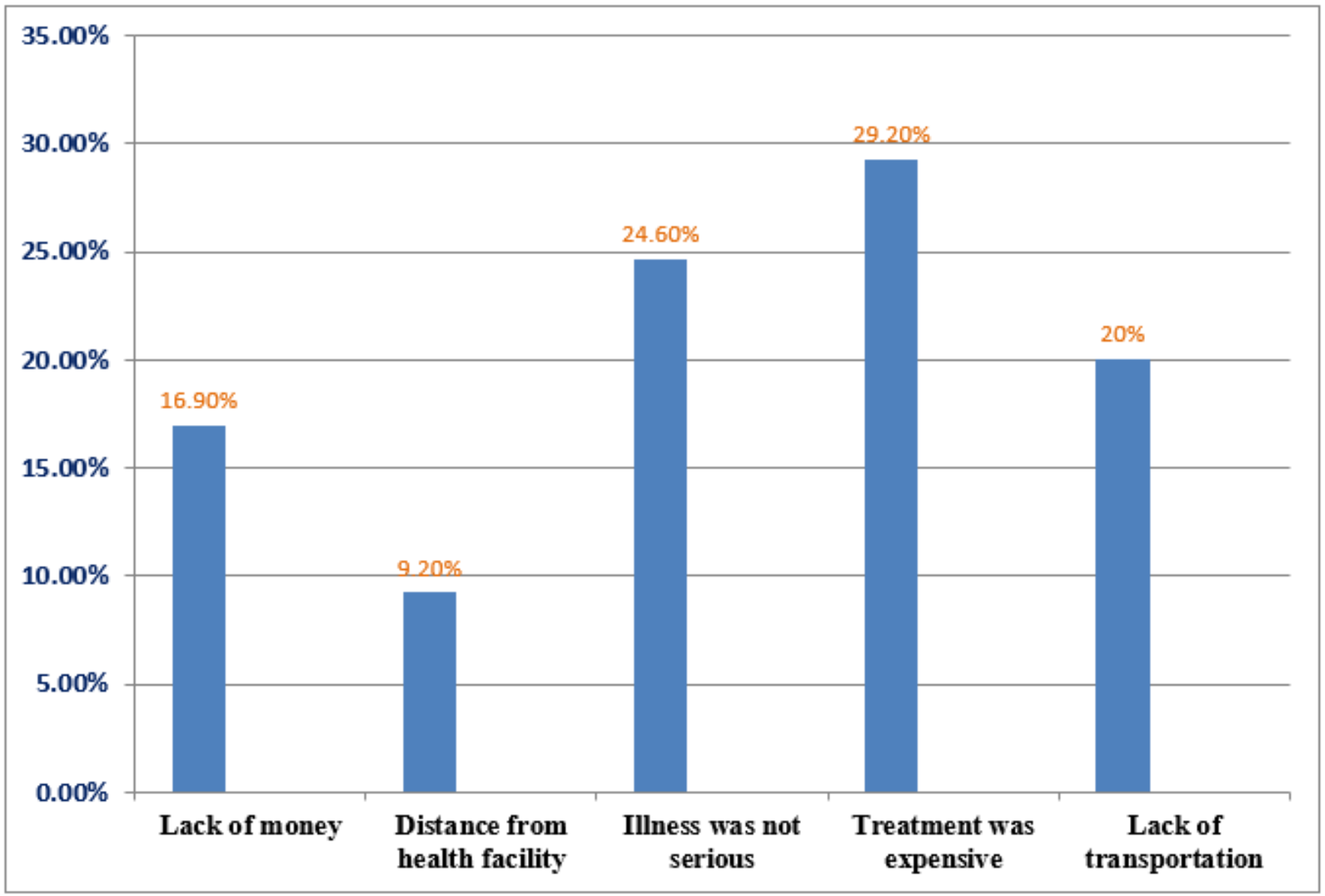


Figure 2

Reasons reported for not visiting health facilities for common childhood illnesses in Efratana Gidim woreda, east Amhara, Ethiopia, 2020. $(n=65)$ 